\title{
New SERS Substrates For Polycyclic Aromatic Hydrocarbon (PAH) Detection: Towards Quantitative SERS Sensors For Environmental Analysis
}

\author{
O. Péron ${ }^{\mathrm{a}, \mathrm{b}}$, E. Rinnert ${ }^{\mathrm{a}}$, C. Compère ${ }^{\mathrm{a}}, \mathrm{T}$. Toury ${ }^{\mathrm{b}}$ \\ and M. Lamy de la Chapelle \\ ${ }^{a}$ Service Interfaces et Capteurs, Département Recherches et Développements Technologiques, IFREMER, \\ BP70, 29280 Plouzané, France. \\ ${ }^{b}$ Laboratoire de Nanotechnologie et d'instrumentation Optique, Institut Charles Delaunay, FRE 2848, \\ Université de technologie de Troyes, 12 rue Marie Curie, 10010 Troyes, France. \\ ${ }^{c}$ Laboratoire CSPBAT (FRE 3043), UFR SMBH, Université Paris XIII, 74 rue Marcel Cachin, 93017 \\ Bobigny, France.
}

\begin{abstract}
In the investigation of chemical pollutions, such as PAHs (Polycyclic Aromatic Hydrocarbons) at low concentration in aqueous medium, surface-enhanced Raman scattering (SERS) stands for an alternative to the inherent low cross-section of normal Raman scattering. Indeed, SERS is a very sensitive spectroscopic technique due to the excitation of the surface plasmon modes of the nanostructured metallic film.
\end{abstract}

Keywords. Quantitative SERS sensors, Polystyrene beads, PAHs, nanoparticles.

The surface of quartz substrates is coated with a hydrophobic film obtained by silanization and subsequently reacted with colloidal suspension of gold metal nanoparticles. The hydrophobic surface of the SERS substrates pre-concentrates non polar molecules such as PAHs. Under laser excitation, the SERS substrates allow the detection and the identification of the target molecule localized close to gold nanoparticles. A limit of detection of $10 \mathrm{ppb}$ has been obtained for naphthalene and pyrene solutions [1,2]. Nonetheless, under excitation the organic part of the active substrate, i.e. the hydrophobic film, seems to keep evolving. Such a drawback can be overcome thanks to hydrophobic SERS substrates based on polystyrene beads surrounded by gold nanoparticles. Furthermore, the Raman fingerprint of the polystyrene stands for a spectral internal reference. To this extent, such SERS substrates could be used as quantitative SERS sensors for environmental analysis.

Within the scope of characterizing the morphology of the SERS substrates, scanning electron microscopy (SEM) measurements were done. Figure 1 (a) shows an overview of the SERS substrate surfaces. The PS beads, with a measured diameter around $800 \mathrm{~nm}$, are deployed onto the substrate surface as a monolayer. The SEM images clearly show that the PS bead surfaces are partially coated; gold nanoparticles were locally grafted to the PS bead surface. Besides gold nanoparticles are also present onto the hydrophobic film. Gold nanoparticles have got an estimated diameter of $\sim 50 \mathrm{~nm}$ (Fig. 1 (b)). Nevertheless, such a gold coating of PS beads is sufficient to generate SERS effect. In order to limit the contribution of the SERS effect due to the gold nanoparticles localized onto the hydrophobic film, all the SERS measurements were performed with the laser beam focused onto the PS beads (Fig. 1 (c)). In the range 1 to 20 ppm naphthalene concentration, a linear fit was plotted (Fig. 1 (d)). In such a range 
of naphthalene concentration, an attractive SERS sensor was obtained. Indeed considering the experimental error bar, the linear fit makes possible a determination of the concentration of a naphthalene solution.

(a)
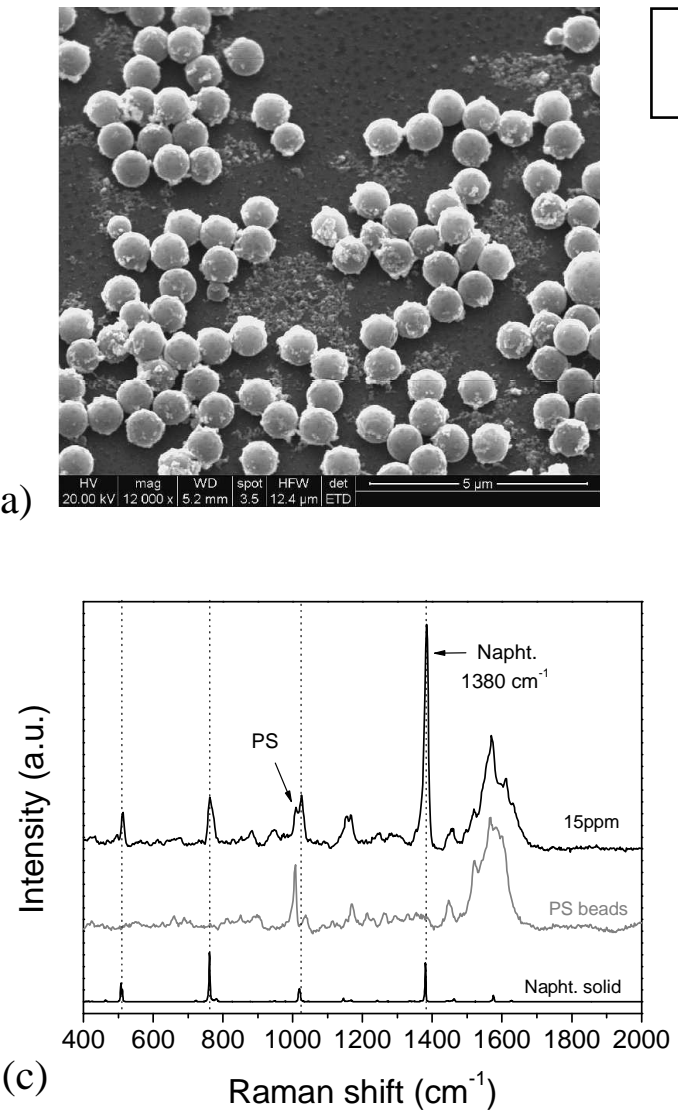

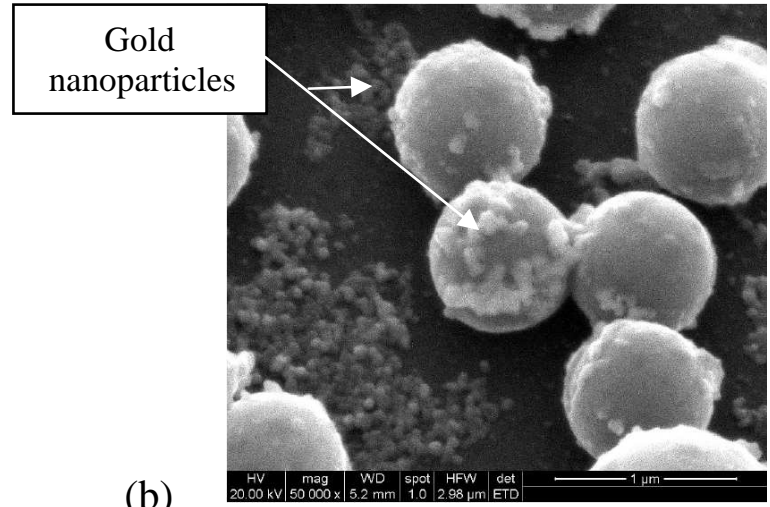

(b)

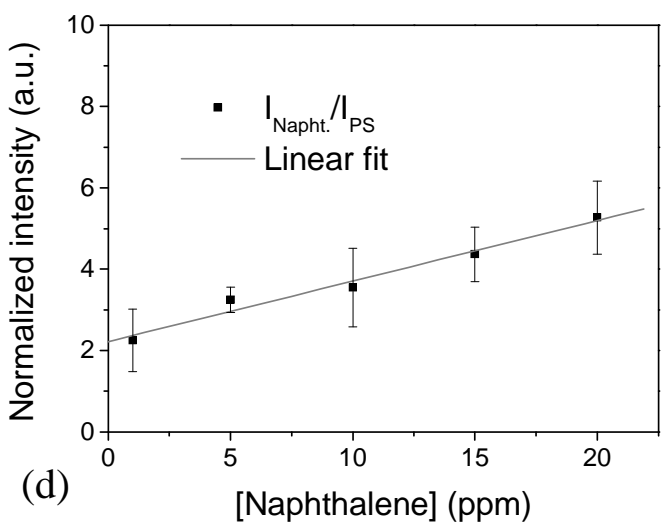

FIGURE 1: SEM images of SERS substrate based on polystyrene beads coated with gold nanoparticles (a) x12 000 and (b) x50 000. (c) SERS spectra of naphthalene solution (15 ppm), PS beads through the blank and for comparison Raman spectrum of solid-state naphthalene is also given. Integration time of $2 \times 10$ s. (d) Normalized intensity $\left(I_{\text {Napht.1380 } \mathrm{cm}^{-1}} / \mathrm{I}_{\mathrm{PS} .1004 \mathrm{~cm}^{-1}}\right)$ versus the naphthalene concentration in a range 1 to $20 \mathrm{ppm}$ and the linear fit. Each point corresponds to the average of 3 or 4 measurements on a same area of the sample. The error bar is calculated as the difference between the maximal and the minimal intensity.

\section{ACKNOWLEDGEMENTS}

This project was supported by ANR financing (P2IC: Discomar) and carried out in conjunction with the Laboratoire de Nanotechnologie et d'Instrumentation Optique (LNIO) - Université de Technologie de Troyes (UTT).

\section{REFERENCES}

1. O. Péron, E. Rinnert, M. Lehaitre, P. Crassous and C. Compère, Talanta 79, 199-204 (2009).

2. O. Péron, E. Rinnert, M. Lehaitre, F. Colas and C. Compère, Proceeding of SPIE Defense, Security \& Sensing 7312, 73120D1-10 (2009). 